\title{
A general form of Drucker-Prager type smooth and convex plastic potential. Part 2: Implementation in elastoplastic damaged material
}

\author{
Inez Kamińska ${ }^{1, *}$ \\ ${ }^{1}$ Warsaw University of Technology, FCE, ul. L. Kaczyńskiego 16, 00-637 Warszawa, Poland
}

\begin{abstract}
The new potential proposed in [1] is used to modify Concrete Damaged Plasticity [2] model. The formulas for plastic multiplier and elastoplastic stiffness tensor are derived and simple numerical test are performed to confirm validity of the change. Predictions of the modified model and the original model are compared. The comparison shows similar character of the resultant curves, although for some cases a distinct quantitative difference between the models is revealed.
\end{abstract}

\section{Introduction}

The main goal of this paper is to confirm that the potential proposed in Part I [1] is valid for concrete. For this purpose, Concrete Damaged Plasticity model available in Abaqus [2] is modified - the new function is introduced in the yield condition instead of Lubliner function [3]. As proven in the preceding article, the considered function is differentiable for all stress states, which allows to derive elastoplastic modulus tensor for damaged material in a concise way.

The Lubliner function is appropriate for describing behaviour of concrete for low and moderate values of hydrostatic stress $|\xi|$, which is usually the area of interest in civil engineering applications. The yield surface based on the proposed potential is similar to the surface introduced by Lubliner, but two significant disadvantages are removed. Lubliner's function is non-smooth on the compression meridians and on the curve, where one of the principal stresses is null, which is inconvenient during calculations. Moreover, the plane stress cross-section does not comply well with experimental results. The new yield surface does not have any singularities and the cross-section fits in with empirical data.

The considered material model describes elastoplastic behaviour with isotropic damage. In Part I, the proposed potential was calibrated on the basis of results of four tests and the coefficients were tied to the yield limit for uniaxial compression test $\sigma_{C}$. To use that result, only isotropic hardening is considered.

In Section 2, the general structure of the model is presented, along with the elastoplastic stiffness tensor for damaged material. In Section 3, results of numerical computations for

*Corresponding author: ikam@il.pw.edu.pl 
several tests for concrete are shown along with comparison to the original model predictions.

\section{Formulation of model}

\subsection{Assumptions}

Concrete Damaged Plasticity (CDP) [2] model allows to describe elastoplastic behaviour of damaged material. The following modification of the model is proposed: Lubliner yield condition is replaced with the new potential [1] in order to improve compatibility of numerical calculations with experimental results. The structure of the model is presented below.

In the model, damage is represented by one scalar variable $0 \leq d \leq 1$ [4]. Effective (actual) stress $\hat{\boldsymbol{\sigma}}$ (the stress for undamaged material) and nominal stress $\boldsymbol{\sigma}$ (for the damaged material) are related as follows:

$$
(1-d) \hat{\boldsymbol{\sigma}}=\boldsymbol{\sigma},
$$

where the hypothesis of equivalent strain is used [5].

Additive split of strain rate tensor $\dot{\boldsymbol{\varepsilon}}$ into elastic, $\dot{\boldsymbol{\varepsilon}}_{E}$, and plastic, $\dot{\boldsymbol{\varepsilon}}_{P}$, parts holds, which together with Hooke's law yield the following:

$$
\hat{\boldsymbol{\sigma}}=\mathbf{C} \cdot \boldsymbol{\varepsilon}_{E} \quad \Rightarrow \quad \dot{\hat{\boldsymbol{\sigma}}}=\mathbf{C} \cdot\left(\dot{\boldsymbol{\varepsilon}}-\dot{\boldsymbol{\varepsilon}}_{P}\right), \quad \text { where } \quad \mathbf{C}=K \mathbf{I} \otimes \mathbf{I}+2 G\left(\mathbf{1}-\frac{1}{3} \mathbf{I} \otimes \mathbf{I}\right)
$$

is the stiffness tensor for isotropic undamaged material.

Plasticity and damage occur simultaneously - the yield condition is identical with the damage criterion. While for plastic flow the damage parameter increases, for unloading it remains constant, reducing elastic stiffness of the material. The constitutive relations for plasticity are formulated in the effective stress space. The yield condition and the consistency condition employ proposed potential $f[1]$ :

$$
f(\hat{\xi}, \hat{r}, \hat{\theta})=0 \quad \text { and } \quad \dot{f}(\hat{\xi}, \hat{r}, \hat{\theta})=0 .
$$

The non-associated flow rule is based on the Drucker-Prager type potential:

$$
\dot{\boldsymbol{\varepsilon}}_{P}=\lambda \frac{\partial \Phi}{\partial \hat{\boldsymbol{\sigma}}}=\lambda \mathbf{p}, \quad \text { where: } \quad \Phi(\hat{\xi}, \hat{r})=\sqrt{\left(\hat{\sigma}_{T} \beta_{M} \operatorname{tg} \Psi\right)^{2}+\frac{3}{2} \hat{r}^{2}}+\frac{1}{\sqrt{3}} \operatorname{tg} \Psi \hat{\xi}
$$

and $\hat{\sigma}_{T}$ is the effective plastic limit for uniaxial tension test, $\beta_{M}$ is the eccentricity and $\Psi$ is the dilation angle [2].

Damage parameter $d$ allows to distinguish compressive and tensile stress states by using two components: $d_{T}$ and $d_{C}$ dependent on inelastic equivalent tensile strain $\varepsilon_{T, I N}$ and inelastic equivalent compressive strain $\varepsilon_{C, I N}$ respectively:

$$
1-d=\left(1-s_{T} d_{C}\right)\left(1-s_{C} d_{T}\right), \text { where: } \quad d_{T}=H_{T} \varepsilon_{T, I N}, d_{C}=H_{C} \varepsilon_{C, I N}
$$




$$
\begin{gathered}
\text { and } \varepsilon_{C, I N}=\varepsilon_{C, P L}+\frac{d_{C}}{1-d_{C}} \frac{\sigma_{C}}{E}, \quad \varepsilon_{T, I N}=\varepsilon_{T, P L}+\frac{d_{T}}{1-d_{T}} \frac{\sigma_{T}}{E}, \\
\dot{\varepsilon}_{C, P L}=\left(r^{*}-1\right) \dot{\varepsilon}_{P, M I N}, \quad \dot{\varepsilon}_{T, P L}=r^{*} \dot{\varepsilon}_{P, M I N}, \quad r^{*}=\frac{\left\langle\hat{\sigma}_{1}\right\rangle+\left\langle\hat{\sigma}_{2}\right\rangle+\left\langle\hat{\sigma}_{3}\right\rangle}{\left|\hat{\sigma}_{1}\right|+\left|\hat{\sigma}_{2}\right|+\left|\hat{\sigma}_{3}\right|} .
\end{gathered}
$$

$s_{C}$ and $s_{T}$ are coefficients reflecting material properties and current stress state, while $H_{C}$ and $H_{T}$ are material parameters describing evolution of damage during uniaxial test [2]. $E$ is the initial Young modulus and \langle\rangle is the Macaulay bracket. The evolution of equivalent compressive and tensile plastic strains $\left(\varepsilon_{C, P L}\right.$ and $\left.\varepsilon_{T, P L}\right)$ depends on the minimum and maximum principal values of the strain rate tensor $\left(\dot{\varepsilon}_{P, M I N}\right.$ and $\left.\dot{\varepsilon}_{P, M A X}\right)$ and the current stress state. Coefficient $r^{*}$ determines the degree of tensile behaviour for effective stress tensor. When all the principal effective stresses are positive $\hat{\sigma}_{1} \geq \hat{\sigma}_{2} \geq \hat{\sigma}_{3}>0$ then $r^{*}=1$. If they are all negative $r^{*}=0$.

Plastic hardening occurs only during compression and it is governed by function:

$$
\sigma_{C}=\sigma_{C 0}+E_{P} \varepsilon_{C, I N}
$$

where $\sigma_{C}$ is the current plastic limit for uniaxial compression test, $\sigma_{C 0}$ is the initial yield limit for uniaxial compression test and $E_{P}$ is the plastic modulus.

For the model to be thermodynamically consistent the Clausius-Duhem [6] inequality for dissipation $D$ has to be fulfilled:

$$
D=\hat{\boldsymbol{\sigma}} \cdot \dot{\boldsymbol{\varepsilon}}_{P}+\frac{1}{2} \dot{d}\left(\boldsymbol{\varepsilon}-\boldsymbol{\varepsilon}_{P}\right) \cdot \mathbf{C} \cdot\left(\boldsymbol{\varepsilon}-\boldsymbol{\varepsilon}_{P}\right) \geq 0
$$

Condition (7) has to be checked during numerical simulation.

The presented model contains 18 parameters: 2 material constants for describing elastic behaviour ( $E$ and $v$ or $K$ and $G$ ), 8 for the yield condition $\left(\alpha_{1}, \alpha_{2}, \beta_{1}, \beta_{2}, A, B, b\right.$, $k$ ) and 3 for the flow rule $\left(\beta_{M}, \Psi, \hat{\sigma}_{T}\right)$; plastic modulus $E_{P}$ describing hardening and damage moduli $H_{C}$ and $H_{T}$ along with $s_{C}$ and $s_{T}$.

The proposed yield surface differs from the original one, namely the Lubliner function. As shown in Fig.1, the new function allows for including a shear meridian test into calibration. Also, the meridians are fitted in well with the experimental results. The difference between the yield functions is noticeable when comparing the plane stress crosssections for purely compressive states $\left(\sigma_{1}<0\right.$ and $\left.\sigma_{2}<0\right)$. The new potential is passed through two points representing compression tests (for $\sigma_{1}=\sigma_{2}$ and $\sigma_{1}=0.5 \sigma_{2}$ ), which ensures better compatibility with the experimental results than for the Lubliner function. Furthermore, the curve for the Lubliner yield condition is non-smooth for $\sigma_{1}=-\sigma_{C}$ and $\sigma_{2}=-\sigma_{C}$ (uniaxial compression).

The calibration proposed in [1] is rather simplistic. It is possible to further adjust the coefficients to fit empirical results, especially for the plane stress cross-section, although it requires numerical solution of a set of nonlinear equations. Nonetheless, the new function allows to take into account more characteristic features of concrete's yield surface, which in turn enhances flexibility of the model. 


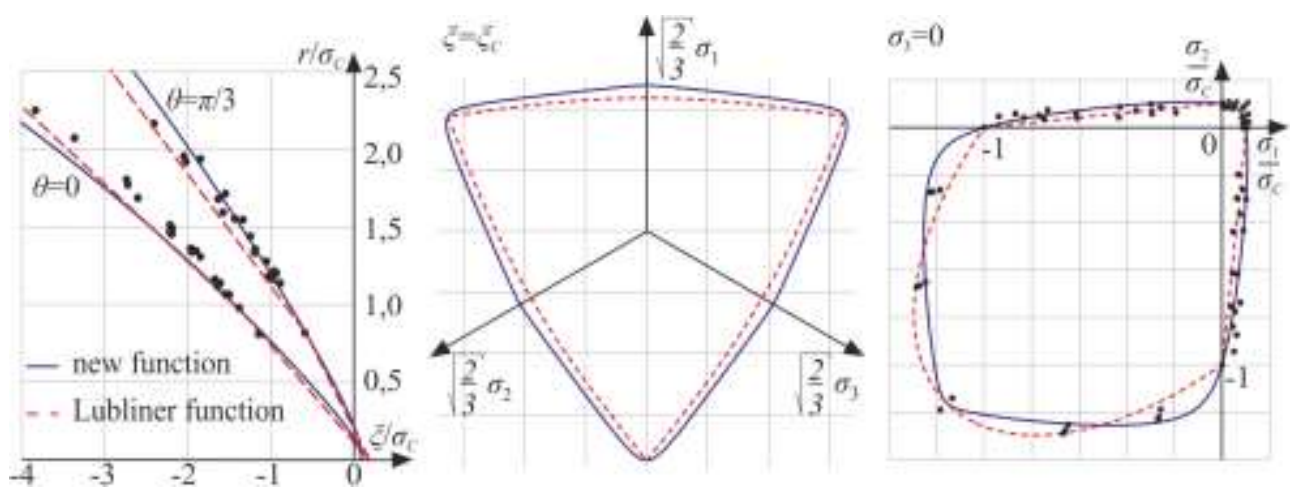

Fig. 1. The new yield surface versus the original yield surface $[2,3]$ and experimental results $[7,8]$.

\subsection{Elastoplastic stiffness tensor}

For practical applications it is convenient to determine the formula for plastic multiplier $\lambda$ and elastoplastic stiffness tensor for undamaged material $\mathbf{C}_{E P}$ and for damaged material $\mathbf{C}_{E P D}$ defined as follows:

$$
\dot{\hat{\boldsymbol{\sigma}}}=\mathbf{C}_{E P} \bullet \dot{\boldsymbol{\varepsilon}} \Rightarrow \dot{\boldsymbol{\sigma}}=(1-d) \mathbf{C}_{E P} \bullet \dot{\boldsymbol{\varepsilon}}=\mathbf{C}_{E P D} \bullet \dot{\boldsymbol{\varepsilon}}
$$

where weak coupling between plasticity and damage is supposed via $\dot{\boldsymbol{\sigma}}=(1-d) \dot{\hat{\boldsymbol{\sigma}}}$.

In order to derive the relations, the standard procedure is followed. The additive split is used for Hooke's law (2) $)_{2}$ and the plastic multiplier is determined from consistency condition $(3)_{2}$ considering flow rule (4) and the evolution laws for equivalent strains and damage parameters. The resultant formulas are listed below:

$$
\begin{gathered}
\lambda=\frac{1}{A_{0}}\left\{\left[3 K U_{1}+\left(K-\frac{2}{3} G\right) U_{3} \hat{r}^{2}\right] \dot{\boldsymbol{\varepsilon}}+2 G\left(U_{2} \hat{\mathbf{s}} \bullet \dot{\boldsymbol{\varepsilon}}+U_{3} \hat{\mathbf{s}}^{2} \cdot \dot{\boldsymbol{\varepsilon}}\right)\right\} \\
\mathbf{C}_{E P}=2 G \mathbf{1}+\left\{K-\frac{2}{3} G-\frac{1}{A_{0}}\left[3 K U_{1}+\left(K-\frac{2}{3} G\right) U_{3} \hat{r}^{2}\right] K \operatorname{tg} \psi\right\} \mathbf{I} \otimes \mathbf{I}+ \\
-\frac{1}{A_{0}} 2 K G \operatorname{tg} \psi\left(U_{2} \mathbf{I} \otimes \hat{\mathbf{s}}+U_{3} \mathbf{I} \otimes \hat{\mathbf{s}}^{2}\right)+ \\
\frac{3 G}{A_{0} \sqrt{\left(\hat{\sigma}_{T} \beta_{M} \operatorname{tg} \psi\right)^{2}+\frac{3}{2} \hat{r}^{2}}}\left\{\left[3 K U_{1}+\left(K-\frac{2}{3} G\right) U_{3} \hat{r}^{2}\right] \hat{\mathbf{s}} \otimes \mathbf{I}+2 G U_{2} \hat{\mathbf{s}} \otimes \hat{\mathbf{s}}+2 G U_{3} \hat{\mathbf{s}} \otimes \hat{\mathbf{s}}^{2}\right\}
\end{gathered}
$$




$$
\begin{aligned}
& \text { where: } U_{2}=\left\{H\left\{1-\frac{b \cos 3 \hat{\theta} \operatorname{tg}\left[\frac{1}{3} \arccos (b \cos 3 \hat{\theta})-k\right]}{\sqrt{1-(b \cos 3 \hat{\theta})^{2}}}\right\} \text { for } 0<\theta<\frac{\pi}{3}\right. \text {, } \\
& \text { H } \\
& \text { for } \theta=0 \text { or } \theta=\frac{\pi}{3} \text {, } \\
& U_{3}=\left\{\begin{array}{cl}
\frac{\sqrt{6} b H \operatorname{tg}\left[\frac{1}{3} \arccos (b \cos 3 \hat{\theta})-k\right]}{\hat{r} \sqrt{1-(b \cos 3 \hat{\theta})^{2}}} & \text { for } 0<\theta<\frac{\pi}{3}, \\
0 & \text { for } \theta=0 \text { or } \theta=\frac{\pi}{3},
\end{array}\right. \\
& H=\frac{\alpha_{1} f_{2 N}+\alpha_{2} f_{1 N}-\left(\alpha_{1}+\alpha_{2}\right) F}{\left(f_{1 N}+f_{2 N}-2 F\right) g \sqrt{(A g)^{2}+\hat{r}^{2}}}, \\
& A_{0}=\left(3 U_{1}+U_{3} \hat{r}^{2}\right) K \operatorname{tg} \psi+\frac{3 G \hat{r}^{2}\left(U_{2}+\frac{1}{\sqrt{6}} U_{3} \hat{r} \cos 3 \hat{\theta}\right)}{\sqrt{\left(\hat{\sigma}_{T} \beta_{M} \operatorname{tg} \psi\right)^{2}+\frac{3}{2} \hat{r}^{2}}}+h_{1} E_{P}\left(1-r^{*}\right) p_{M I N}, \\
& =\frac{\frac{\left(\alpha_{1} f_{2 N}+\alpha_{2} f_{1 N}-\left(\alpha_{1}+\alpha_{2}\right) F\right) A \frac{\mathrm{d} A}{\mathrm{~d} \sigma_{C}} g}{\sqrt{(A g)^{2}+\hat{r}^{2}}}+\frac{B}{2} \frac{\mathrm{d} B}{\mathrm{~d} \sigma_{C}}+\left(F-f_{2 N}\right) \frac{\mathrm{d} \beta_{1}}{\mathrm{~d} \sigma_{C}}+\left(F-f_{1 N}\right) \frac{\mathrm{d} \beta_{2}}{\mathrm{~d} \sigma_{C}}}{f_{1 N}+f_{2 N}-2 F},
\end{aligned}
$$

where $p_{\text {MIN }}$ is the minimum eigenvalue of $\mathbf{p}$. On the tension and compression meridians it is necessary to separately define the selected functions defined above because of different form of some derivatives for $\theta=0$ and $\theta=\pi / 3$ in Haigh-Westergaard coordinate system (see [9] for details).

\section{Simple computational tests}

In this Section simple tests are performed to confirm the validity of the enhanced model and to examine the differences to the original one. To achieve this goal, an elementary algorithm is created using Mathematica [10] where the explicit Euler scheme is exploited. The results of uniaxial compression test, biaxial compression test with $\sigma_{1}=\sigma_{2}$ and shear test $\left(\sigma_{1}=-\sigma_{2}\right)$ are shown.

In all computations the following values are used: $E=20 \mathrm{GPa}$ and $v=0.2$ $(K=11 \mathrm{GPa} \quad$ and $\quad G=8.3 \mathrm{GPa}) ; \quad \sigma_{C 0}=23 \mathrm{MPa}, \quad \sigma_{T 0}=0.1 \sigma_{C 0}, \quad \sigma_{B C 0}=1.16 \sigma_{C 0}$, $\sigma_{B N C 0}=1.25 \sigma_{C 0}$ and $\sigma_{T C 0}=1.25 \sigma_{C 0}$ with $\eta=4.91$, which yields: $\alpha=0.121, \gamma=2.370$ and $\beta=7.667$ for the Lubliner function and $\alpha_{1}=0.86424, \alpha_{2}=5.8336, \beta_{1}=3.7630 \sigma_{C}$, $\beta_{2}=20.462 \sigma_{C}, A=0.14187 \sigma_{C}, B=16.651 \sigma_{C}, b=0.98270$ and $k=0.10489$ for the new potential (with $d=2.75 \sigma_{C}$ ); for the flow rule: $\beta_{M}=0.1, \phi=30^{\circ}$. The parameters 
describing hardening and damage are: $E_{P}=5 \mathrm{GPa}, H_{C}=120, H_{T}=120, \omega_{C}=0.1$, $\omega_{T}=1[2]$.

For the improved model, a uniaxial cyclic compression test is carried out with loading program shown in Fig. 2a. Damage and plasticity occur simultanously, therefore damage parameter $d$ increases only during plastic flow (Figure $2 \mathrm{~b}$ ). For the considered test the growth is approximately linear, although it is nonlinear in general. The value of axial component of total strain $\varepsilon_{X X}$ is considerably higher than the lateral component $\varepsilon_{Y Y}$, but the respective components of plastic strain are comparable (Figure 2c). For proposed data, plastic strain is an essential part of total strain. The relations between strains $\varepsilon_{X X}$ and $\varepsilon_{Y Y}$ and $\sigma_{X X}$ are approximately bilinear (Figure 2d). The results are qualitatively correct.

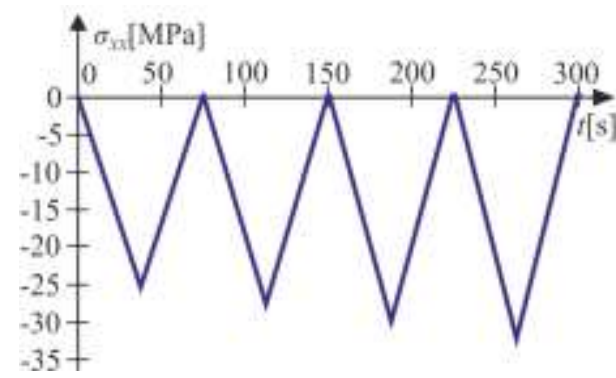

a)

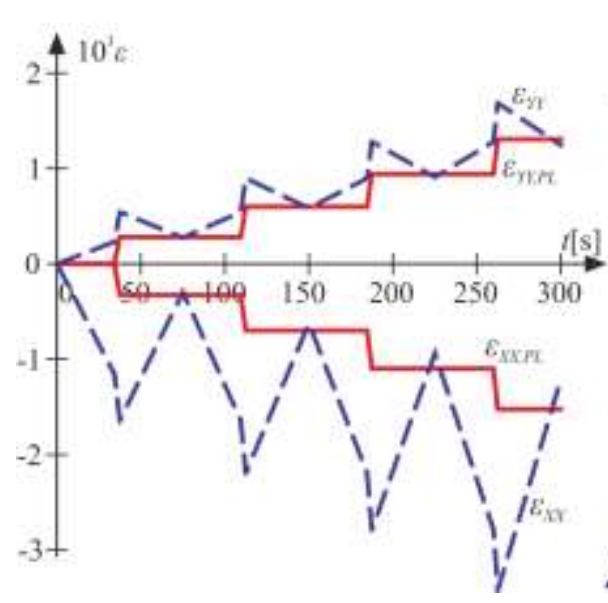

c)

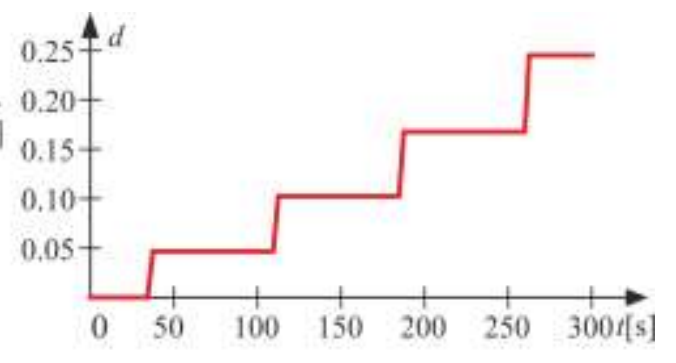

b)

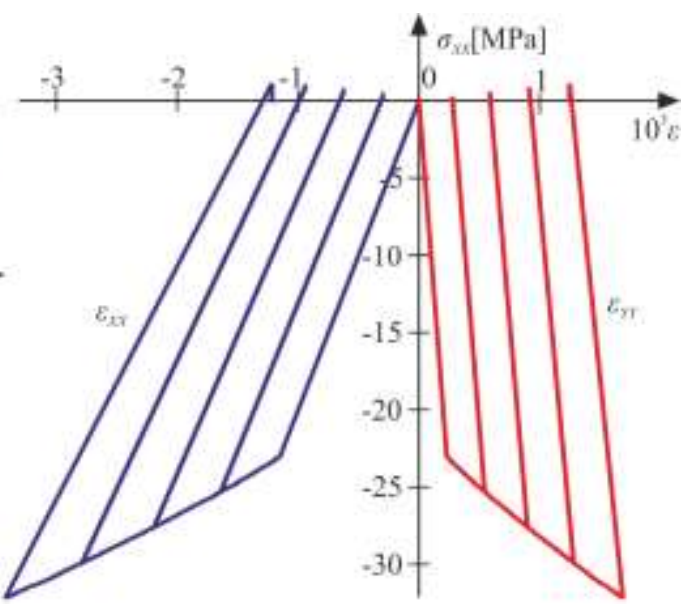

d)

Fig. 2. Uniaxial cyclic compression test for the improved model.

To notice the influence of the change of the yield condition three test are compared: uniaxial compression test, uniaxial tension test and simple shear test, located on different meridians: $\theta=\pi / 3, \theta=0$ and $\theta=\pi / 6$, respectively. The loading program for the indicated stress components is:

$$
\sigma(t)=\frac{-2 \sigma_{C 0}}{t_{M A X}} t, \quad \text { where } \quad t_{M A X}=300 \mathrm{~s}
$$


is the duration period of the analysis and for the three mentioned test the stress tensors are as follows:

$$
\boldsymbol{\sigma} \rightarrow\left[\begin{array}{ccc}
\sigma(t) & 0 & 0 \\
0 & 0 & 0 \\
0 & 0 & 0
\end{array}\right], \boldsymbol{\sigma} \rightarrow\left[\begin{array}{ccc}
\sigma(t) & 0 & 0 \\
0 & \sigma(t) & 0 \\
0 & 0 & 0
\end{array}\right] \text { and } \boldsymbol{\sigma} \rightarrow\left[\begin{array}{ccc}
0 & \sigma(t) & 0 \\
\sigma(t) & 0 & 0 \\
0 & 0 & 0
\end{array}\right]
$$

The selected results of the computations are presented in Fig. 3. The curves obtained for the original and the modified model are similar, both qualitatively and quantitatively. It is apparent, that using the proposed function smaller values of components of plastic strain tensor are obtained for uniaxial compression test and biaxial compression test, which delays the moment of total failure $(d=1)$. The discrepancy between the models is especially noticeable on the shear meridian (Figure 3c), where the plastic limits for simple shear predicted by the new and original function vary substantially.
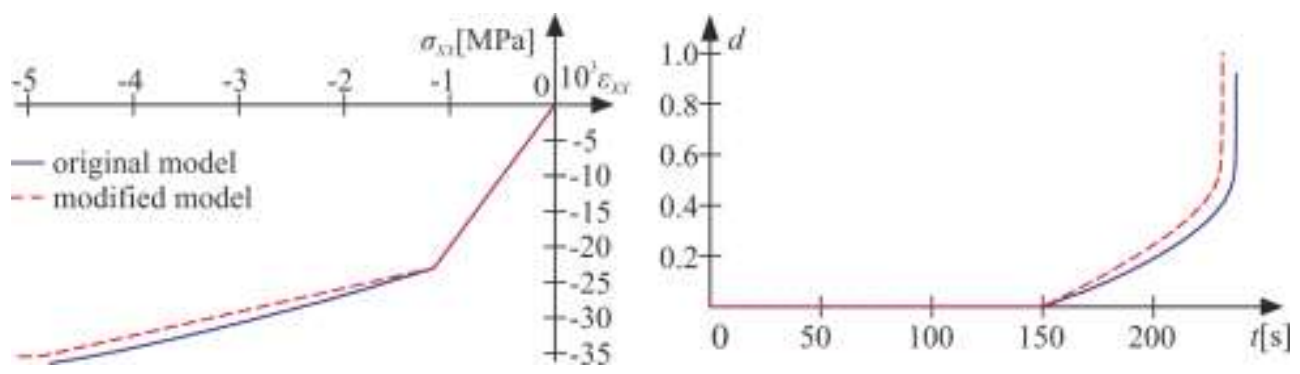

a) uniaxial compression test
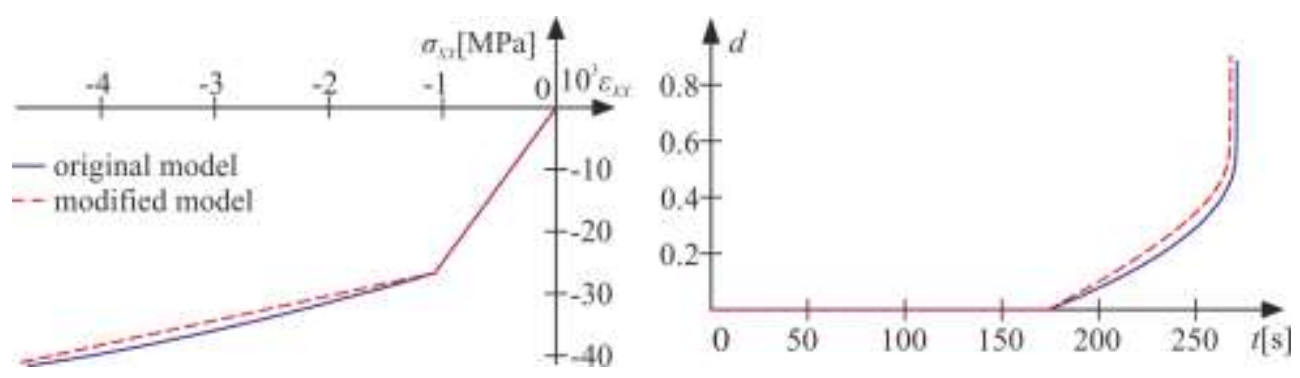

b) biaxial compression test
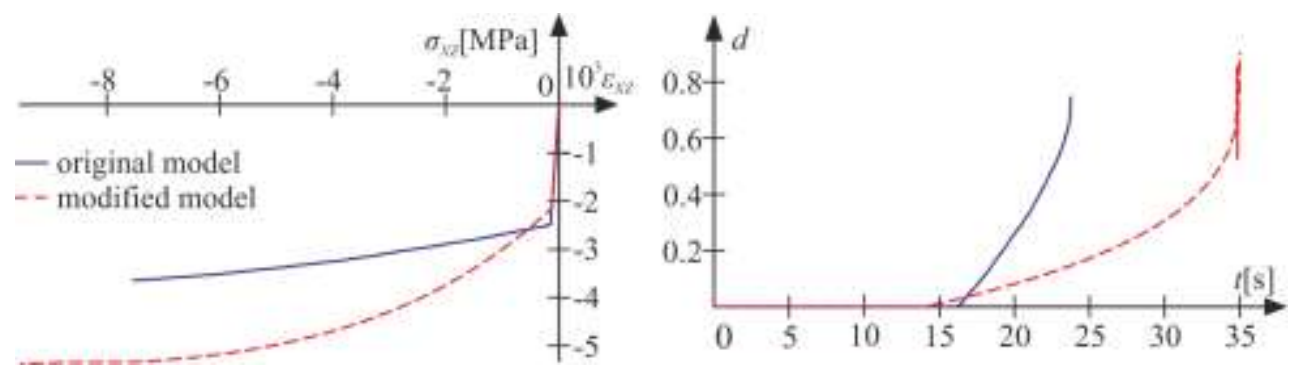

c) shear test

Fig. 3. Uniaxial compression test, biaxial compression test and simple shear test - comparison of the models' predictions. 


\section{Conclusions}

The performed calculations confirm validity of the model modified with the new potential proposed in [1] showing qualitative adequacy of the results. The proposed yield condition extends flexibility of the model, permitting to describe more of characteristic features in material behaviour through introduction of a number of parameters.

The calibration of the yield condition was shown in Part I [1], but to be able to compare the results of numerical tests with empirical data, calibration of the whole model needs to be performed. Especially the determination of the hardening curve is of major importance, which in this paper is assumed in a very simple form.

Developing yield conditions to better fit experimental data is reasonable only when the proposed functions are complex enough to enrich the description of material, whereas sufficiently simple to allow to find their coefficients. The proposed potential has both these advantages and is additionally smooth and convex which simplifies numerical calculations. It can be succesfully used as a yield condition as well as a plastic potential for the flow rule.

\section{References}

1. A. Szwed, I. Kamińska, Part I - accompanying paper

2. ABAQUS: Theory Manual, Version 6.3 (Hibbit, Karlson \& Sorensen Inc., 2002)

3. J. Lubliner, J. Oliver, S. Oller, E. Onate, Int J Solids Struct, 25(3), 299-326 (1989)

4. L.M. Kachanov, Introduction to continuum damage mechanics (Springer, 1896)

5. S. Murakami, Continuum Damage Mechanics (Springer, 2012)

6. N.S. Ottosen, M. Ristinmaa, The Mechanics of Constitutive Modeling (Elsevier, 2005)

7. L. Mills, M. Zimmerman, ACI J, 67(10), 802-807 (1970)

8. H. Kupfer, H. Hilsdorf, H. Rusch, ACI J, 66(8), 656-666 (1969)

9. D. Bigoni, A. Piccolroaz, Int J Solids Struct, 41, 2855-2878 (2004)

10. www.wolfram.com 